\title{
Structure of Human BCCIP and Implications for Binding and Modification of Partner Proteins
}

\author{
Woo Suk Choi ${ }^{1}$, Bochao Liu², Zhiyuan Shen², Wei Yang ${ }^{1}$ \\ ${ }^{1}$ Laboratory of Molecular Biology, NIDDK, National Institutes of Health, \\ 9000 Rockville Pike, Building 5, Bethesda, MD 20892, USA \\ ${ }^{2}$ Rutgers Cancer Institute of New Jersey, Department of Radiation Oncology, Rutgers \\ Robert Wood Johnson Medical School, 195 Little Albany Street, New Brunswick, NJ \\ 08901
}

Corresponding author: Wei Yang (weiy@niddk.nih.gov)

Zhiyuan Shen (shenzh@cinj.rutgers.edu)

Running title:

Keywords:

Abbreviations:
Crystal structure of human $\mathrm{BCCIP} \beta$

BRCA2, tumor suppressor, ribosome biogenesis, acetyltransferase 
Choi et al., 2020

\begin{abstract}
BCCIP was isolated based on its interactions with tumor suppressors BRCA2 and p21. Knockdown or knockout of BCCIP causes embryonic lethality in mice. BCCIP deficient cells exhibit impaired cell proliferation and chromosome instability. BCCIP also plays a key role in biogenesis of ribosome 605 subunits. BCCIP is conserved from yeast to humans, but it has no discernible sequence similarity to proteins of known structures. Here we report two crystal structures of an $\mathrm{N}$-terminal truncated human BCCIP $\beta$, consisting of residues 61-314. Structurally BCCIP is similar to GCN5-related acetyltransferases (GNATs) but contains different sequence motifs. Moreover, both acetyl-CoA and substrate-binding grooves are altered in BCCIP. A large 19-residue flap over the putative CoA binding site adopts either an open or closed conformation in BCCIP. The substrate binding groove is significantly reduced in size and is positively charged despite the acidic isoelectric point of BCCIP. BCCIP has potential binding sites for partner proteins and may have enzymatic activity.
\end{abstract}


Choi et al., 2020

\section{INTRODUCTION}

BCCIP is a nuclear protein that was identified in human genome based on its interactions with tumor suppressors BRCA2 and p21 1, 2. In humans, there are two isoforms resulting from alternative RNA splicing, $\mathrm{BCCIP} \alpha$ (322 residues) and $\mathrm{BCCIP} \beta$ (314 residues), which share the identical $\mathrm{N}$-terminal 258 residues but differ in the remaining C-terminal regions ${ }^{1,2}$. BCCIP $\alpha$ and $\beta$ are also known as TOK-1 $\alpha$ and TOK$1 \beta$, respectively ${ }^{1}$. BCCIP $\beta$ is the conserved isoform in eukaryotes, from yeast, worms, plants to mammals ${ }^{2}$, while BCCIP $\alpha$ only exists in humans. In mouse, there is only one BCCIP, which is $\sim 70 \%$ identical to human BCCIP $\beta$. Either knockdown or knockout of BCCIP in mice leads to embryonic lethality due to impaired cell proliferation ${ }^{3,4}$. BCCIP deficient mouse embryo fibroblast cells exhibit increased sensitivity to DNA damage and replication stress and increased chromosome instability, including chromosome breaks and sister chromatid union ${ }^{3}$. The yeast homolog of $B C C I P \beta$, known as $B C P 1$, appears to be involved in nuclear export and ribosome biogenesis ${ }^{5,6}$. Recently, both mouse BCCIP and human BCCIP $\beta$ were shown to be located in the nucleolus and required for rRNA maturation and ribosome 605 subunit biogenesis ${ }^{7}$, but with distinct features from the yeast $\mathrm{BCP} 1$.

Despite its functional importance, structures of BCCIP-family proteins remain elusive. Except for limited sequence similarity of $\sim 50$ residues to the $\mathrm{Ca}^{2+-b i n d i n g ~ d o m a i n ~}$ in calmodulin and M-calpain ${ }^{2}$, BCCIP has no discernible sequence homology to any known protein. Using isomorphous replacement we have determined crystal structures of a large fragment of human BCCIP $\beta$ (aa 61-314) in two different crystal forms. To our 
surprise, structurally BCCIP is homologous to the GCN5-related N-acetyltransferases (GNATs) ${ }^{8}$, which use acetyl-CoA to modify primary amines of lysine sidechains or protein $\mathrm{N}$ termini, as well as aminoglycosides (antibiotics) and hormones (serotonin) ${ }^{9-12}$. The regions corresponding to the substrate and catalytic motifs in $\mathrm{N}$-acetyltransferases (motifs A-D), are also conserved among BCCIP-family members but are different from GNATs. Whether BCCIP is an enzyme, and what its substrates might be are unclear. While we were engaged in trying to figure out its structure-function relationship, a yeast BCP1 structure was reported earlier this year (with the structure coordinates on hold) ${ }^{13}$. Here we present crystal structures of the conserved isoform of human BCCIP $\beta$ and its outstanding binding surfaces for partner proteins and possibly for small molecules.

\section{RESULTS AND DISCUSSION}

\subsection{Crystal structures of human BCCIP}

We have determined crystal structures of $\mathrm{N}$-terminal truncated BCCIP $\beta$ (aa 61-314, referred to as BCCIP hereafter) in two different space groups at resolutions of 3.06 (Native1) and $2.13 \AA$ (Native2), respectively (Table 1). The two structures are superimposable except for a 19-residue extended and flexible loop. BCCIP forms a single domain of $\alpha / \beta$ fold, including a mixed seven-stranded $\beta$ sheet surrounded on either side by four and three long $\alpha$ helices (Fig. 1). The structure can be divided into two halves. The N-terminal half (aa 61-185) folds into two $\beta \alpha \alpha \beta$ hairpins with the four $\beta$ strands ( $\beta 1$ $\beta 4)$ forming an antiparallel sheet, and four $\alpha$ helices forming two hairpins $(\alpha A-\alpha B$, and $\alpha \mathrm{C}-\alpha \mathrm{D}$ ) covering one face of the $\beta$ sheet. Helix $\alpha \mathrm{C}$ is preceded by a short helix $\alpha \mathrm{C}^{\prime}$ 
(aa132-136). The C-terminal half (aa 186-314) folds in the order of $\alpha \beta \alpha \beta \beta \alpha$ and forms a three-stranded antiparallel $\beta$ sheet ( $\beta 5-7)$, with three $\alpha$ helices $(\alpha E-\alpha G)$ on the side opposite to helices $\alpha A-\alpha D$ (Fig. 1a-b). The two halves are linked by helix $\alpha E$ and form a contiguous $\beta$ sheet by hydrogen bonds at the beginning of parallel strands $\beta 4$ and $\beta 5$. These two strands diverge at the end giving the $\beta$ sheet a $V$ shape (Fig. 1b). No $\mathrm{Ca}^{2+}$ binding module or metal ion-binding site can be found. The extended loop linking $\beta 6$ and $\beta 7, L_{67}$ (aa 269-287), has two dramatically different conformations. In Native1 $L_{67}$ is extended and interacts with a crystallographic symmetry mate reciprocally. But in Native2 $L_{67}$ is folded next to $\alpha A-\alpha B$, and $L_{67}$ and $\alpha A B$ are like two arms embracing the $\beta$ sheet (Fig. 1b). The $\mathrm{N}$-terminal half followed by $\alpha E$ and $\beta 5$ has the same sequence in the two isoforms of human BCCIP, and only $\alpha \mathrm{F}-\beta 6-\beta 7-\alpha \mathrm{G}$ differ between the two.

\subsection{BCCIP is structurally similar to GNAT acetyltransferases}

Structural similarity search by Dali revealed that BCCIP is similar to GCN5-related acetyltransferases (GNATs), with substrates ranging from histones to antibiotics and hormones. The N-terminal half of BCCIP is superimposable with these GNATs (Fig. 2a). But in GNATs helices $\alpha \mathrm{C}$ and $\alpha \mathrm{D}$ are absent and $\beta 3$ and $\beta 4$ are linked by a $\beta$ hairpin directly or a random coil $9-12,14$. Helices $\alpha \mathrm{C}$ and $\alpha \mathrm{D}$ effectively close the open binding groove for protein substrates, such as the histone tail bound to GCN5 ${ }^{14}$, and fill it with hydrophobic residues. With its positively charged pocket (Fig. 1c) BCCIP may still be able to bind small molecules, such as antibiotics and hormones. 
The structurally most conserved region (motifs $A$ and $B$ ) involving in acetyl-CoA binding in GNATs is located between two halves of the structure at $\beta 4-\beta 5^{8}$. The corresponding region is also conserved among BCCIP-family members, but the sequence motifs of BCCIP (Fig. 1b) differ from GNATs ${ }^{15}$.

The C-terminal half of BCCIP is oriented differently from GNATs, particularly loop $L_{5 F}$, which binds the diphosphates of CoA in GNATs. BCCIP contains an insertion in $L_{5 F}$, and without structural changes, its $L_{5 F}$ would clash with the CoA moiety Fig. $2 b$ ). In case substrate binding induces conformational changes in BCCIP, we tried co-crystallization of BCCIP with 10 different acyl-CoA compounds. Instead of finding a bound substrate, we obtained a different form of apo BCCIP crystal (Native2) (see section 3.2).

In addition, compared to GNATs, BCCIP has the following two insertions, the extended flexible loop L67, which is open and extended in Native1 and adopts a closed conformation in Native2 (Fig. 2c), and the C-terminal helix $\alpha$ G. Together with $L_{5 F}$, these insertions alter the acetyl-CoA binding site significantly. Peculiarly, two Tyr residues, Y64 and $\mathrm{Y} 71$, are exposed

\subsection{Potential binding surface for protein and small molecules}

The conserved residues among BCCIP homologs are clustered into two groups. One is around $\beta 1-\alpha \mathrm{A}$ and the loop linking $\beta 2$ and $\beta 3$, forming a predominantly negatively charged convex surface (Fig. 1c). Peculiarly two conserved Tyr, Y64 and Y71, are solvent exposed and begging for an interacting partner. The other is between $\beta 4$ and $\beta 5$, the $\mathrm{V}$ shaped substrate-binding groove and pocket (top and front in Fig. 1c). Aromatic residues 
also abound the second cluster. Surrounding the V-shaped binding groove, two disordered loops $L_{23}$ (aa 110-119) and $L_{5 F}$ (aa 229-244) are next to the flexible flap $L_{67}$ (Fig. 1a-b), like tentacles waiting to embrace a catch. Human, mouse and yeast BCCIPs are overall negatively charged with isoelectric point below 4.8. However, substrate binding groove and pocket have a positively charged appearance, but they are surrounded by an intensely negatively charged belt (Fig. 1c). These features indicate that BCCIP binds specific protein partners and possibly a small molecule substrate in the same location as GNATs.

\section{MATERIALS AND METHODS}

\subsection{BCCIP expression and purification}

The N-terminal 60 residues of BCCIP $\beta$ were predicted to be highly disordered, and probably prevented crystallization of the full-length BCCIP $\beta$ (data not shown). The Nterminal truncation construct of BCCIP $\beta$ (aa 61-314, abbreviated as BCCIP) was cloned into pET-28c(+) (Novagene Corp. Inc.) between Ncol and EcoRI sites, and the resulting plasmid was transformed into BL21 (DE3) competent cells. The cells were grown in LB medium at $37^{\circ} \mathrm{C}$ until $\mathrm{OD}_{600}$ reached $\sim 0.6$. After cooling down to $16^{\circ} \mathrm{C}$ for $30 \mathrm{~min}$, expression of BCCIP was induced by addition of $0.3 \mathrm{mM} \mathrm{IPTG}$ at $16^{\circ} \mathrm{C}$ for 20 hours. The cells were pelleted, re-suspended in buffer $\mathrm{A}(20 \mathrm{mM}$ sodium phosphate $(\mathrm{pH} 7.4), 500 \mathrm{mM}$ $\mathrm{NaCl}$ ) with $40 \mathrm{mM}$ imidazole, and lysed by sonication. The lysate was cleared by centrifugation at $30,000 \mathrm{~g}$ for 1 hour at $4^{\circ} \mathrm{C}$. BCCIP was bound to an $\mathrm{Ni}^{2+}$ column and eluted in buffer A plus $300 \mathrm{mM}$ imidazole. The eluted protein was further purified using a Hitrap Q HP column (GE Healthcare) equilibrated in buffer B (25mM Tris- $\mathrm{HCl}(\mathrm{pH} 7.5)$, 
$100 \mathrm{mM} \mathrm{NaCl}, 1 \mathrm{mM}$ EDTA, 0.01\% IGEPAL-CA630, and $1 \mathrm{mM} \mathrm{DTT}$ ) and was eluted with a $\mathrm{NaCl}$ gradient from 100 to $1000 \mathrm{mM}$. As a final step, BCCIP was applied to a Hiprep Sephacryl S-200 16/60 column (GE healthcare) equilibrated in buffer B. The BCCIP peak was pooled and concentrated to $\sim 15 \mathrm{mg} / \mathrm{ml}$ and stored at $-80^{\circ} \mathrm{C}$ in buffer $\mathrm{B}$ and $50 \%$ glycerol.

\subsection{Crystallization}

BCCIP was buffer exchanged to $25 \mathrm{mM}$ Tris- $\mathrm{HCl}(\mathrm{pH} 7.5)$ and $1 \mathrm{mM}$ DTT in an Amicon Ultra-0.5 (Millipore, 10k cutoff) and concentrated to $15 \mathrm{mg} / \mathrm{ml}(514 \mu \mathrm{M})$. Crystallization screening was performed at $4^{\circ} \mathrm{C}$ with JCSG core I (Qiagen) using the sitting drop vapor diffusion method. The protein and precipitant were mixed at equal volume $(0.1 \mu \mathrm{l}$ each). Native1 crystals were obtained in $0.2 \mathrm{M}$ tri-sodium citrate and 1820\% PEG3350. The initial crystals diffracted X-rays only to $\sim 3.3 \AA$. After improving protein purification and additions of $1 \mathrm{mM} \mathrm{DTT}, 100 \mathrm{mM} \mathrm{NaCl}$ and $50 \%$ glycerol in the protein storage buffer, BCCIP crystals (Native1) became much more reproducible and diffracted X-rays to nearly $3.0 \AA$.

After realizing that BCCIP is similar to GNATs, we tested co-crystallization of BCCIP with various acyl-CoA compounds ${ }^{16}$. Among 10 acyl-CoAs we tried, crystals grown with $1.1 \mathrm{mM}$ benzoyl(bz)-CoA with a precipitant of $0.1 \mathrm{M}$ citric acid ( $\mathrm{pH} 4.5$ ) and 1$2 \%$ PEG 6000 at $20^{\circ} \mathrm{C}$ diffracted X-rays to $2.13 \AA$. But after structural determination by molecular replacement, the resulting electron density map showed no bz-CoA. Bz-CoA thus functioned as an additive in crystallization, and it changed the crystal space group with improved X-ray diffraction, to a form we termed Native2 (Table 1). 
The BCCIP crystals were transferred into the respective stabilization solutions (Native1 in $0.2 \mathrm{M}$ tri-sodium citrate (pH 8.0), 25\% PEG 3350, and $1 \mathrm{mM}$ DTT, Native2 in $0.1 \mathrm{M}$ citric acid ( $\mathrm{pH} 4.5$ ), 5\% PEG 6000, and $1 \mathrm{mM} \mathrm{DTT)} \mathrm{for} 30 \mathrm{~min}$ before freezing. Native2 crystals cracked into smaller pieces in the stabilization solution. BCCIP crystals were cryo-protected by $20 \%$ (Native1) or $3 \%$ (Native2) ethylene glycol and flash-frozen in liquid nitrogen.

\subsection{Phase determination}

We selected twelve commonly used $\mathrm{Hg}$ (mercury), $\mathrm{Pt}$ (platinum), $\mathrm{Au}$ (gold) and $\mathrm{Pb}$ (lead) compounds, each of which was tested in $0.2 \mathrm{mM}$ or $5 \mathrm{mM}$ concentration, and soaked into the crystals for 1 to 24 hours. We monitored crystal morphology, birefringence and X-ray diffraction at various time intervals. Before flash-freezing in liquid nitrogen, crystals were washed in the stabilization buffer for $15-20$ second. Riso between a heavyatom soaked and native crystal was calculated using Scaleit implemented in CCP4i ${ }^{17,} 18$ to evaluate whether a derivative was made. We determined the BCCIP structure with phases obtained from six datasets of ethylmercuri-thiosalicylic acid derivative (3.15-4.2 Å, Table 1).

\subsection{Data collection and structure determination}

All X-ray data were collected at beamline 22ID (Native1) and 22BM (Native2 and Hg-derivative) at the Advanced Photon Source (APS) at Argonne National Laboratory. Each dataset was processed using HKL2000 (Native1 and Hg derivative) ${ }^{19}$ and XDS (Native2) ${ }^{20}$. Native1 crystals were in $\mathrm{P}_{4} 1_{12}$ space group with one molecule per 
asymmetric unit, and Native2 crystals were in $\mathrm{P} 2{ }_{1}$ space group with three molecules per asymmetric unit (Table 1). For SAD (Single-wavelength Anomalous Dispersion) phasing, $6 \mathrm{Hg}$-derivative datasets were merged to enhance the anomalous signal using HKL2000 at 3.6 Å resolution (Table 1) ${ }^{19}$, and phases were calculated using AutoSol in Phenix ${ }^{21}$. The electron density map was further improved by phase extension to $3.04 \AA$ with the native data. An initial model was built using AutoBuild in PHENIX ${ }^{21}$. Because diffraction of Native1 crystals was highly anisotropic, we corrected the data scaling and truncation using Diffraction Anisotropy Server ${ }^{22}$ to improve the map and refinement. Aided by the secondary structure prediction by PsiPred ${ }^{23}$ we manually traced 228 out of 254 residues using COOT ${ }^{24}$ and refined the BCCIP structure using PHENIX ${ }^{21}$. (Table 1). Two loops linking $\beta 2$ to $\beta 3$ ( $\mathrm{L}_{23}$, aa $\left.110-119\right)$ and $\beta 5$ to $\alpha \mathrm{F}$ ( $\mathrm{L}_{5 \mathrm{~F}}$, aa 229-244) were disordered (Fig. 1).

Native2 structure was determined by molecular replacement using Phaser in PHENIX ${ }^{21}$ with Native1 BCCIP structure as the search model. Model building and refinement were carried out as described above, and non-crystallographic symmetry constraints were applied. Except for the re-orientation of the long loop L67 (aa 269-287) due to different crystal lattice contacts, the two BCCIP structures were superimposable (Fig. 1).

All structure figures were prepared with PYMOL (www.pymol.org). 
Table 1 Statistics of crystallographic data collection and structure refinement

\begin{tabular}{|c|c|c|c|}
\hline Crystal & Hg derivative & Native1 & Native2 \\
\hline PDB & & $7 \mathrm{KYQ}$ & $7 \mathrm{KYS}$ \\
\hline Space group & $P 4,2,2$ & $P 4_{1} 2{ }_{1} 2$ & $P 2_{1}$ \\
\hline \multicolumn{4}{|l|}{ Unit cell } \\
\hline$a, b, c(\AA)$ & $114.4,114.4,53.9$ & $112.7,112.7,56.4$ & $60.0,67.0,99.5$ \\
\hline$a, \beta, \gamma\left(^{\circ}\right)$ & $90.0,90.0,90.0$ & $90.0,90.0,90.0$ & $90.0,91.0,90.0$ \\
\hline Resolution $(\AA)^{2}$ a & $40.0-3.62(3.68-3.62)$ & $40.0-3.04(3.09-3.04)$ & $30.0-2.13(2.19-2.13)$ \\
\hline Wavelength $(\AA)$ & 1.0000 & 1.0000 & 1.0000 \\
\hline$R_{\text {merge }}(\%)$ a & $11.0(86.7)$ & $10.7(98.4)$ & $9.1(157.9)$ \\
\hline$I / \sigma a$ & $22(17)$ & $26(2.3)$ & $7.8(1.0)$ \\
\hline Completeness (\%) a & $100.0(100.0)$ & $99.8(99.4)$ & $98.9(99.9)$ \\
\hline Multiplicity a & $20.2(21)$ & $12.4(12.1)$ & $3.7(3.6)$ \\
\hline \multicolumn{4}{|l|}{ Refinement } \\
\hline Resolution $(\AA ̊)^{\text {a }}$ & & $39.8-3.06$ & $29.7-2.13$ \\
\hline No. reflections & & 6877 & 43893 \\
\hline No. reflections for $R_{\text {free }}$ & & 367 & 1001 \\
\hline$R_{\text {work }} / R_{\text {free }}$ & & $0.219 / 0.251$ & $0.209 / 0.234$ \\
\hline \multicolumn{4}{|l|}{ No. atoms } \\
\hline Overall & & 1894 & 5767 \\
\hline Protein & & 1850 & 5490 \\
\hline Ligand & & - & 39 \\
\hline Water & & 44 & 238 \\
\hline \multicolumn{4}{|l|}{ Mean B-factors } \\
\hline Overall & & 86.8 & 69.5 \\
\hline Protein & & 86.9 & 69.5 \\
\hline Ligand & & - & 76.3 \\
\hline Water & & 84.1 & 69.2 \\
\hline \multicolumn{4}{|l|}{ R.M.S. deviations } \\
\hline Bond lengths $(\AA)$ & & 0.013 & 0.009 \\
\hline Bond angles $\left({ }^{\circ}\right)$ & & 1.539 & 1.262 \\
\hline \multicolumn{4}{|l|}{ Ramachandran } \\
\hline Favored (\%) & & 95.54 & 99.4 \\
\hline Allowed (\%) & & 4.46 & 0.6 \\
\hline Outliers (\%) & & 0 & 0 \\
\hline
\end{tabular}

a Values in parenthesis are of the highest resolution shell. 


\section{FIGURES}

(a)

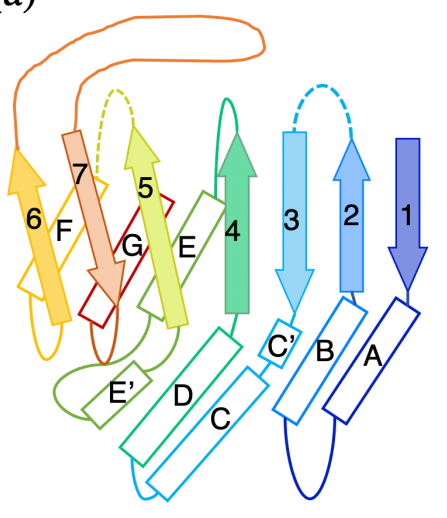

(b)

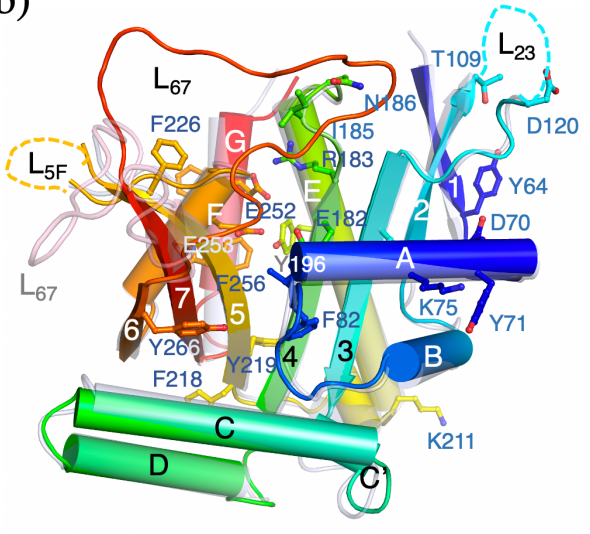

(c) Parner

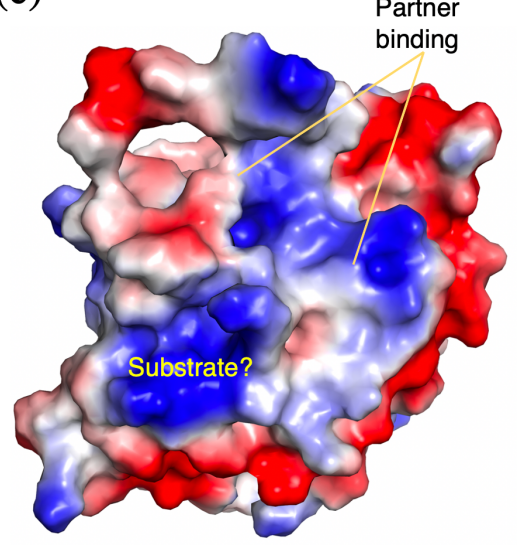

Figure 1 Crystal structures of BCCIP. (a) Topology diagram of BCCIP. The disordered loops are indicated by dashed lines. (b) Cartoon diagrams of superimposed Native1 (semi-transparent grey and pink) and Native2 (solid rainbow colors) structure of BCCIP. Conserved residues are shown as sticks and labeled. (c) Molecular surface of BCCIP (Native2) with electrostatic potential in the same view as in panel b. The potential substrate-binding pocket and interface for protein partners are indicated. 
(a)

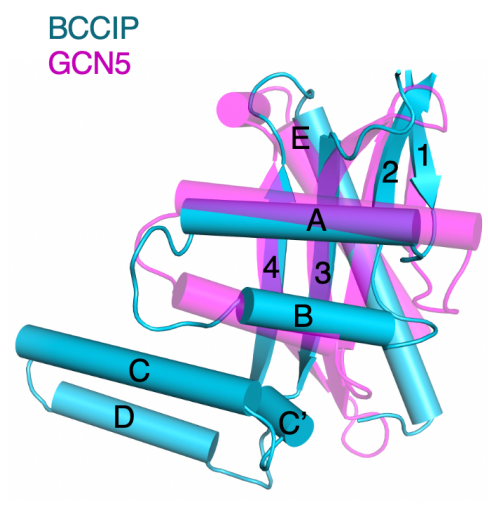

(b)

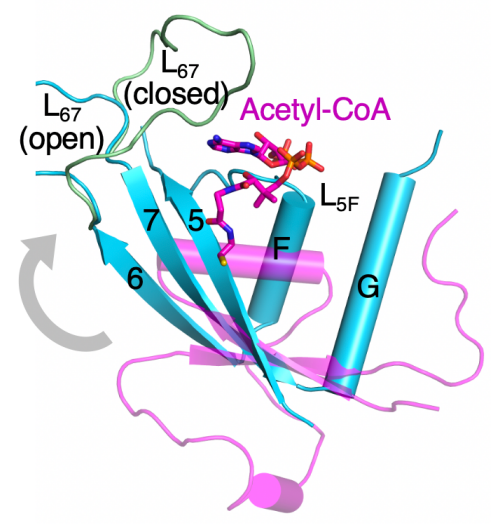

(c)

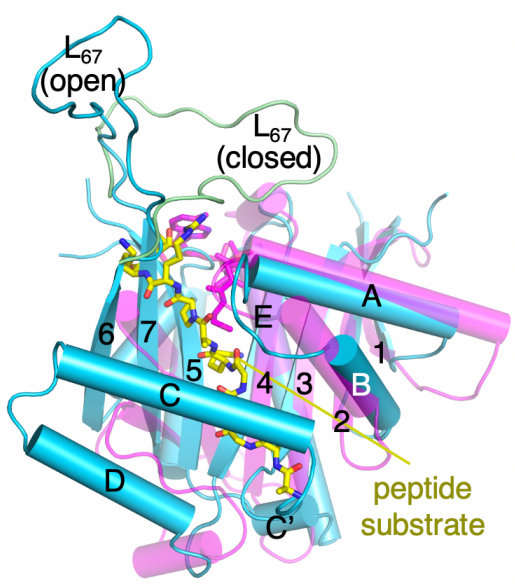

Figure 2 Structural comparison with GCN5. (a) Superposition of the N-terminal half of BCCIP and GCN5 (PDB: 1QSN). (b) After superimposing the N-terminal half, the Cterminal half of BCCIP appears rotated by over $90^{\circ}$ (indicated by the grey arrowhead) relative to that of GCN5. Loop L $_{5 F}$ in BCCIP would clash with acetyl-CoA (bound to GCN5) if present. (c) The entire BCCIP structure is superimposed with GCN5 via the N-terminal half. Histone peptide bound to GCN5 is shown in a yellow (carbon) stick model.

\section{ACKNOWLEDGEMENTS}

The authors thank Dr. M. Gellert for critical reading of the manuscript. The research was supported by the NIH intramural research funding to W. Y. (DK036146) and NIH grant (R01CA195612) to Z.S.

\section{AUTHOR CONTRIBUTIONS}

Bochao Liu: BCCIP preparation and initial crystallization screen. Woo Suk Choi: Crystallization, structure determination, analysis and validation, and manuscript preparation. Wei Yang: structure analysis, figure preparation and writing the original draft. Zhiyuan Shen: Conceptualization; investigation, writing and editing.

\section{CONFLICT OF INTEREST}

The authors declare no potential conflict of interest. 


\section{REFERENCES}

1. Ono, T., et al., TOK-1, a novel p21Cip1-binding protein that cooperatively enhances p21-dependent inhibitory activity toward CDK2 kinase. J Biol Chem, 2000; 275(40): p. 31145-54.

2. Liu, J., et al., Inhibition of breast and brain cancer cell growth by BCCIPalpha, an evolutionarily conserved nuclear protein that interacts with BRCA2. Oncogene, 2001; 20(3): p. 336-45.

3. Lu, H., et al., Essential roles of BCCIP in mouse embryonic development and structural stability of chromosomes. PLoS Genet, 2011; 7(9): p. e1002291.

4. Lu, H., et al., Spontaneous Development of Hepatocellular Carcinoma and B-Cell Lymphoma in Mosaic and Heterozygous Brca2 and Cdkn1a Interacting Protein Knockout Mice. Am J Pathol, 2020; 190(6): p. 1175-1187.

5. Audhya, A. and S.D. Emr, Regulation of PI4,5P2 synthesis by nuclear-cytoplasmic shuttling of the Mss4 lipid kinase. EMBO J, 2003; 22(16): p. 4223-36.

6. $\quad \mathrm{Li}, \mathrm{Z}$., et al., Rational extension of the ribosome biogenesis pathway using networkguided genetics. PLoS Biol, 2009; 7(10): p. e1000213.

7. Ye, C., et al., BCCIP is required for nucleolar recruitment of elF6 and 12S prerRNA production during 60S ribosome biogenesis. Nucleic Acids Res, 2020.

8. Marmorstein, R. and S.Y. Roth, Histone acetyltransferases: function, structure, and catalysis. Curr Opin Genet Dev, 2001; 11(2): p. 155-61.

9. Chang, Y.Y. and C.H. Hsu, Structural basis for substrate-specific acetylation of Nalpha-acetyltransferase Ard1 from Sulfolobus solfataricus. Sci Rep, 2015; 5: p. 8673.

10. Hickman, A.B., et al., The structural basis of ordered substrate binding by serotonin $\mathrm{N}$-acetyltransferase: enzyme complex at $1.8 \mathrm{~A}$ resolution with a bisubstrate analog. Cell, 1999; 97(3): p. 361-9.

11. Wolf, E., et al., X-ray crystallographic studies of serotonin $\mathrm{N}$-acetyltransferase catalysis and inhibition. J Mol Biol, 2002; 317(2): p. 215-24.

12. Stogios, P.J., et al., Structural and Biochemical Characterization of Acinetobacter spp. Aminoglycoside Acetyltransferases Highlights Functional and Evolutionary Variation among Antibiotic Resistance Enzymes. ACS Infect Dis, 2017; 3(2): p. 132-143.

13. Lin, M.H., et al., The crystal structure of protein-transporting chaperone BCP1 from Saccharomyces cerevisiae. J Struct Biol, 2020; 212(1): p. 107605.

14. Rojas, J.R., et al., Structure of Tetrahymena GCN5 bound to coenzyme A and a histone H3 peptide. Nature, 1999; 401(6748): p. 93-8.

15. Neuwald, A.F. and D. Landsman, GCN5-related histone N-acetyltransferases belong to a diverse superfamily that includes the yeast SPT10 protein. Trends Biochem Sci, 1997; 22(5): p. 154-5.

16. Zhao, S., X. Zhang, and H. Li, Beyond histone acetylation-writing and erasing histone acylations. Curr Opin Struct Biol, 2018; 53: p. 169-177.

17. Winn, M.D., et al., Overview of the CCP4 suite and current developments. Acta Crystallogr D Biol Crystallogr, 2011; 67(Pt 4): p. 235-42. 
18. Howell, P.L. and G.D. Smith, Identification of heavy-atom derivatives by normal probablility methods. J. Appl. Cryst., 1992; 25: p. 81-86.

19. Otwinowski, Z. and W. Minor, Processing of X-ray diffraction data collected in oscillation mode. Methods Enzymol, 1997; 276: p. 307-26.

20. Kabsch, W., Integration, scaling, space-group assignment and post-refinement. Acta Crystallogr D Biol Crystallogr, 2010; 66(Pt 2): p. 133-44.

21. Adams, P.D., et al., PHENIX: a comprehensive Python-based system for macromolecular structure solution. Acta Crystallogr D Biol Crystallogr, 2010; 66(Pt 2): p. 213-21.

22. Strong, M., et al., Toward the structural genomics of complexes: crystal structure of a PE/PPE protein complex from Mycobacterium tuberculosis. Proc Natl Acad Sci U S A, 2006; 103(21): p. 8060-5.

23. Buchan, D.W.A. and D.T. Jones, The PSIPRED Protein Analysis Workbench: 20 years on. Nucleic Acids Res, 2019; 47(W1): p. W402-W407.

24. Emsley, P., et al., Features and development of Coot. Acta Crystallogr D Biol Crystallogr, 2010; 66(Pt 4): p. 486-501. 